\title{
Neuro-ophthalmologic manifestations of cholangiocarcinoma: a case series
}

G Rico', SV Smith², Y Siddiqui ${ }^{3}$, A Whyte ${ }^{4}$,

D Gombos $3,4,5$ and AG Lee $2,3,5,6,7,8$

\begin{abstract}
Purpose To describe the neuroophthalmologic findings of cholangiocarcinoma.

Methods We report a retrospective chart review of cholangiocarcinoma patients presenting at two tertiary care centers in the Texas Medical Center.

Results Five patients with neuroophthalmologic symptoms related to cholangiocarcinoma were identified. One patient presented with diplopia due to metastasis to the left medial rectus muscle, two patients had metastasis to the occipital lobe resulting in homonymous hemianopsia, one patient had involvement of the clivus resulting in sixth nerve palsy, and one presented with a hypercoagulable staterelated stroke causing a homonymous hemianopsia and visual hallucinations. Conclusions Neuro-ophthalmic manifestations of cholangiocarcinoma depend upon both mechanism and localization. We report five cases of cholangiocarcinoma with neuroophthalmologic findings. To our knowledge, this is the largest such series reported in the English language ophthalmic literature.
\end{abstract} Eye (2017) 31, 1245-1248; doi:10.1038/eye.2017.77; published online 12 May 2017

\footnotetext{
Introduction

Cholangiocarcinoma is a rare liver malignancy originating in the biliary epithelium. ${ }^{1,2}$ It is uncommon for this malignancy to spread distally, and our review of the literature identified 16 prior cases with brain metastasis and two with orbit metastasis. 3,4 To our knowledge, this is the first and largest series of the neuro-ophthalmologic manifestations of cholangiocarcinoma.
}

\section{Case reports}

\section{Case 1}

A 57-year-old woman with a 4-year history of cholangiocarcinoma experienced loss of vision in the left eye (OS) and gradual worsening diplopia over a 1-month period. Her vision was $20 / 200$ OS with a relative afferent pupillary defect OS and an abduction deficit OS. The right eye exam was normal. Computed tomography (CT) and magnetic resonance imaging (MRI) of the brain and orbit showed an enhancing mass centered within the left medial rectus measuring $2 \mathrm{~cm}$ that extended into the left orbital apex with mild mass effect on the left optic nerve (Figure 1). An orbital biopsy was performed and showed features consistent with the patient's primary cholangiocarcinoma.

\section{Case 2}

A 72-year-old man presented after a syncopal event and the development of a right homonymous hemianopsia. He was found to have multiple cerebral infarcts including the left occipital lobe. An extensive stroke and hypercoagulability workup was performed, revealing a lower extremity deep vein thrombosis, pulmonary artery embolism, and subclavian vein thrombosis. Abdominal imaging revealed a $7 \mathrm{~cm}$ posterior liver mass, which was biopsied and showed features consistent with cholangiocarcinoma. A presumed diagnosis was made of stroke due to the hypercoagulable state of malignancy.

\section{Case 3}

A 53-year-old man presented with bilateral sequential peripheral visual field loss. Two years prior to this, he underwent extensive workup for right lower extremity weakness that confirmed the diagnosis of metastatic
${ }^{1}$ Department of Pathology and Genomic Medicine, Houston Methodist Hospital, Houston, TX, USA

${ }^{2}$ Department of

Ophthalmology, Blanton Eye Institute, Houston Methodist Hospital, Houston, TX, USA

${ }^{3}$ Department of Ophthalmology, UTMB, Galveston, TX, USA

${ }^{4}$ Department Head and Neck Surgery, UT MD Anderson Cancer Center, Houston, TX, USA

${ }^{5}$ Department of Ophthalmology, Baylor College of Medicine, Houston, TX, USA

${ }^{6}$ Section of Ophthalmology, UT MD Anderson Cancer Center, Houston, TX, USA

${ }^{7}$ Departments of Ophthalmology, Neurology, and Neurosurgery, Weill Cornell Medicine, New York City, NY, USA

${ }^{8}$ Department of Ophthalmology, The University of lowa Hospitals and Clinics, lowa City, IA, USA

Correspondence:

AG Lee, Department of Ophthalmology, Blanton Eye Institute, Houston Methodist Hospital, Scurlock Tower 6560 Fannin Street \#450, Houston, TX 77030, USA

Tel: +1 7134418843 ;

Fax: +1 7137906441 E-mail: aglee@

houstonmethodist.org

Received: 22 July 2016

Accepted in revised form: 19 March 2017

Published online: 12 May 2017

Poster presentation at the 2016 North American NeuroOphthalmology Society Annual Meeting in Tucson, AZ, USA. 
disease to lymph nodes and brain from a primary cholangiocarcinoma. The brain metastasis was treated with resection and stereotactic radiotherapy. On examination, he was found to have a dense right

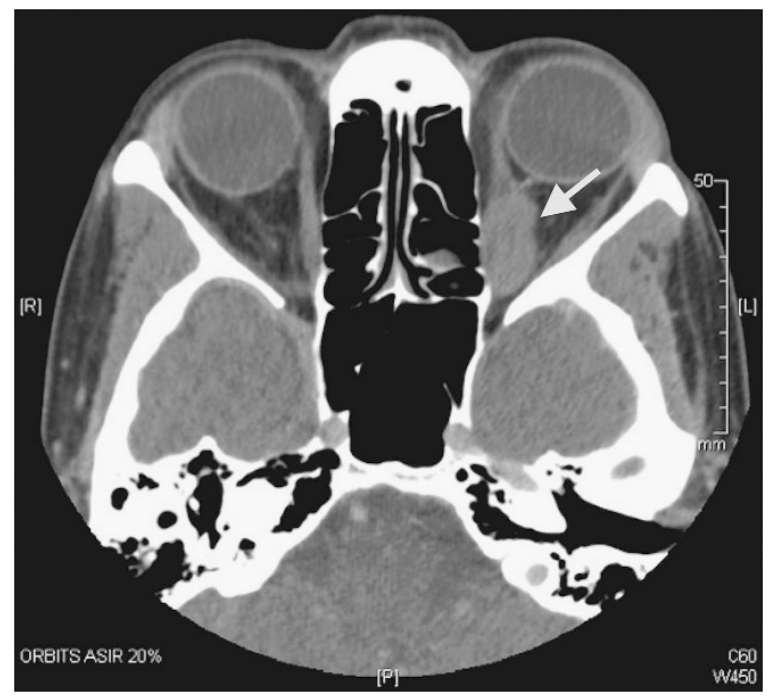

Figure 1 Axial CT scan of the orbits with contrast shows an enhancing mass (arrow) measuring $2 \mathrm{~cm}$ centered in the left medial rectus extending into the left orbital apex. homonymous hemianopsia and a juxtaposed left inferior paracentral macular sparing homonymous quadrantanopsia (Figures 2a and b). A CT scan showed metastatic disease in the left cerebral hemisphere and the right occipital lobe.

\section{Case 4}

A 41-year-old woman presented with a 2-month history of binocular horizontal diplopia. On examination, she had impaired right eye abduction consistent with a right abducens nerve palsy. A brain MRI showed metastasis from a primary cholangiocarcinoma to the skull base involving the clivus and extending beneath the floor of the sella turcica (Figures $3 a$ and b). In addition, fundus examination revealed an elevated amelanotic choroidal lesion in the supra-temporal region of the left eye. Fluorescein angiography and orbital ultrasound were consistent with choroidal metastasis.

\section{Case 5}

A 71-year-old woman with a history of cholagiocarcinoma diagnosed 2 years ago presented with
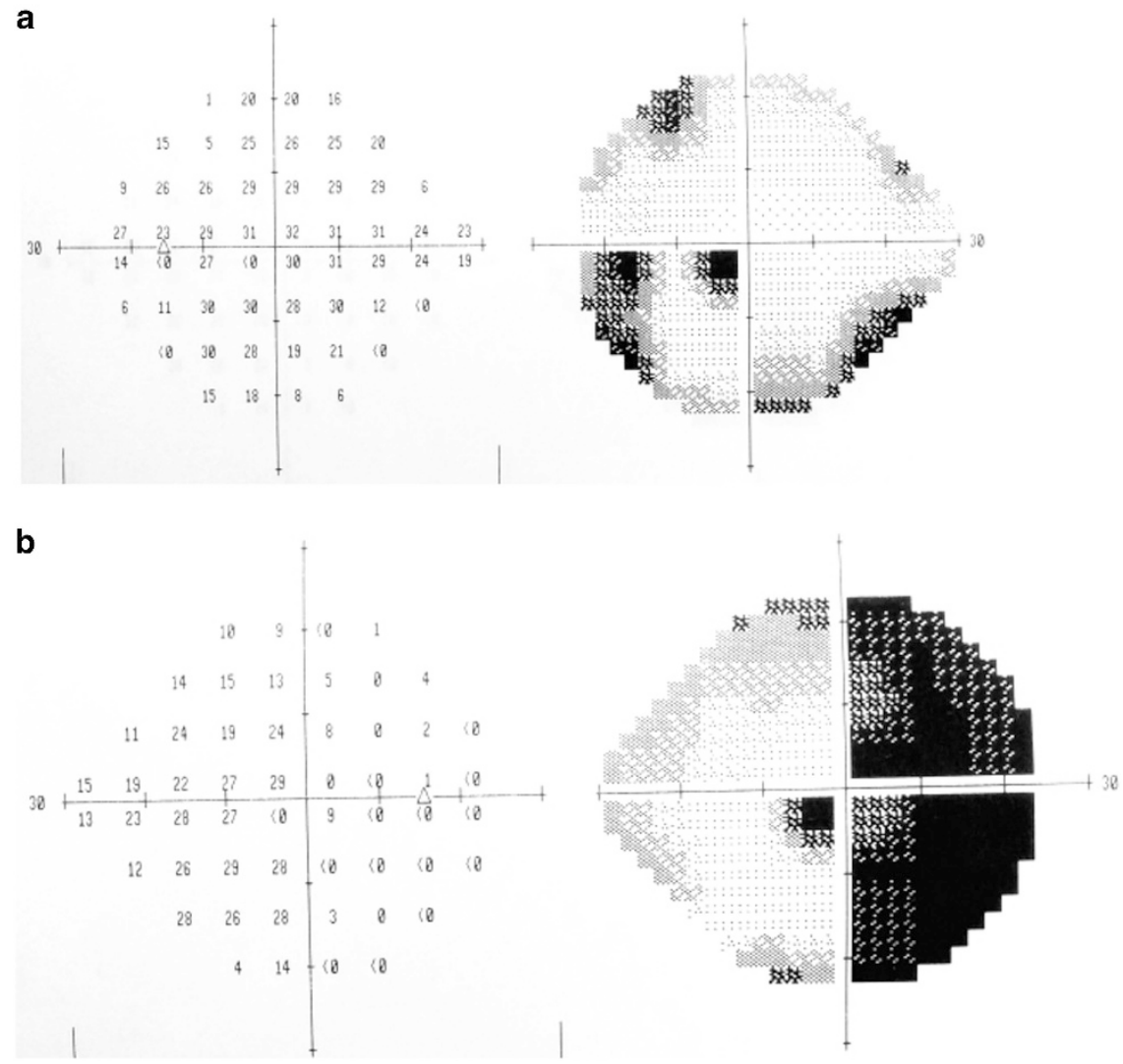

Figure 2 (a) Left eye and (b) right eye: shows a left inferior paracentral homonymous hemianopsia and a juxtaposed, incongruous right homonymous hemianopsia denser in the right eye temporally. 
a

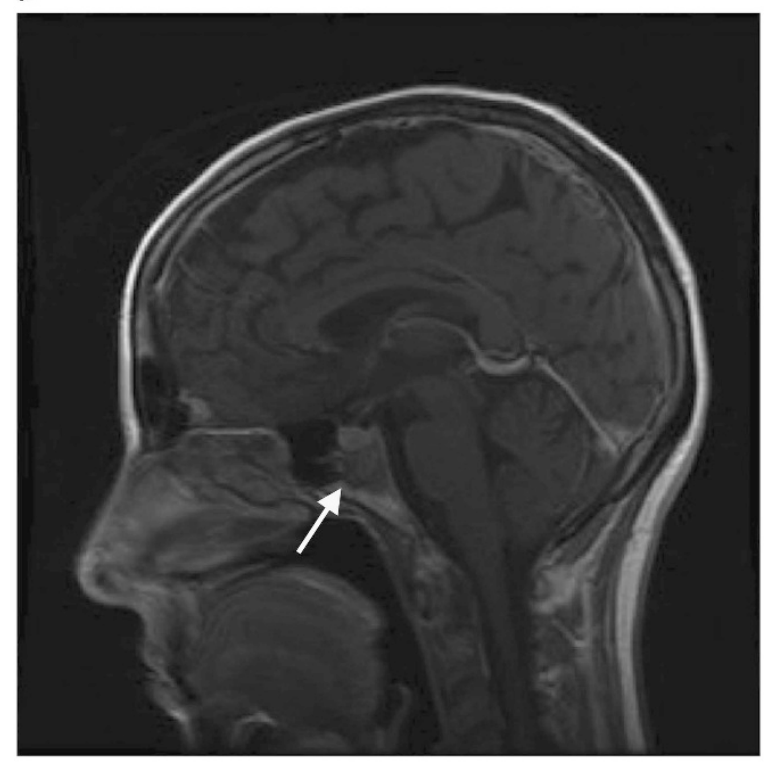

b

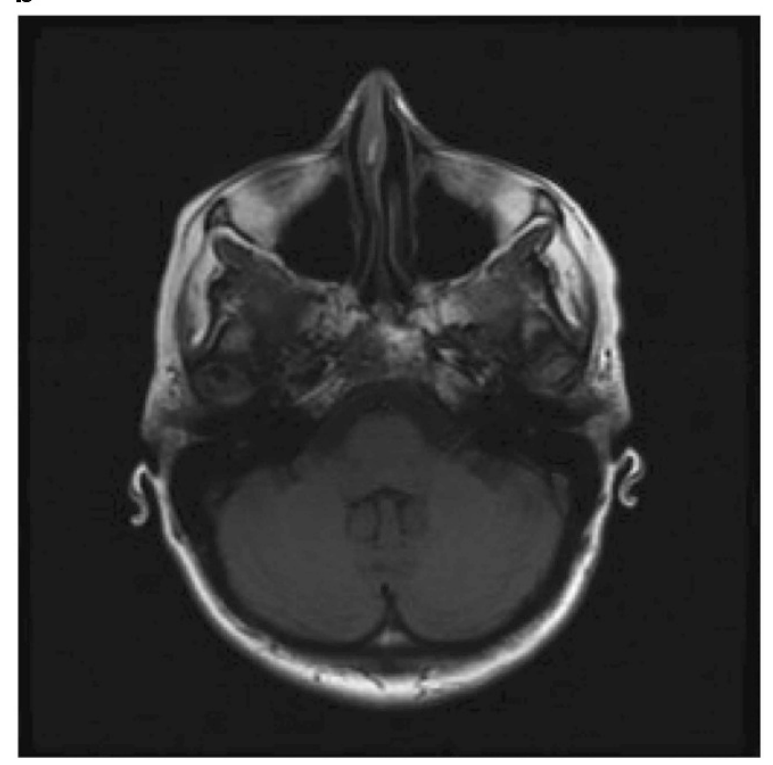

Figure 3 (a) MRI of the brain sagittal view with contrast shows metastasis to the skull base involving the clivus and extending beneath the floor of the sella turcica. (b) Axial view.

acute loss of vision. Examination showed mild cognitive dysfunction, a right homonymous hemianopsia, a few square wave jerks, and an age related supranuclear bilateral reduced upgaze. A cranial CT scan performed 4 months after onset of symptoms revealed a large lesion in the left posterior and occipital region (Figure 4). Tumor resection with pathological analysis confirmed metastatic cholangiocarcinoma. Follow-up brain MRI showed new $3 \mathrm{~mm}$ ring-enhancing nodules along the resection site, suspicious of recurrent disease. Repeat neuroimaging 3 months later continued to show progression in size up

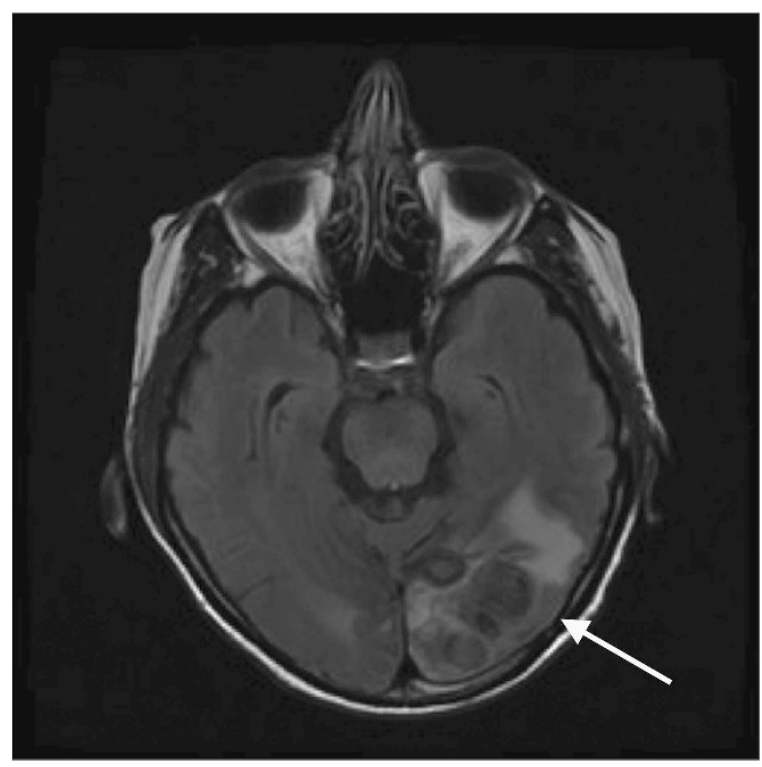

Figure 4 Axial CT scan of the brain showing lesion in the left posterior and occipital region.

to $4.4 \mathrm{~cm}$ with invasion of the adjacent left leaf of tentorium.

\section{Discussion}

Cholangiocarcinoma is a rare malignancy with increasing incidence due to the rise of the common risk factors of obesity and diabetes. ${ }^{4-6}$ It typically presents with abdominal pain, jaundice, and constitutional symptoms. We report five patients who developed neuroophthalmologic manifestations of this rare malignancy. Four patients presented with a known diagnosis of cholangiocarcinoma. One patient however presented with a stroke from the hypercoaguable state of malignancy. In a prior study of 110 patients with cholangiocarcinoma, $47.3 \%$ of patients presented with local disease, $35.5 \%$ had regional (pericholedocal) metastatic disease, and 17.3\% developed distal metastasis. ${ }^{7}$ Scattered case reports have described uncommon sites of metastasis including thyroid, bone, and brain. ${ }^{8-10}$ Brain metastasis typically manifest with headache, motor weakness, and loss of consciousness, ${ }^{11}$ and orbital metastases present with diplopia, proptosis, pain, and decreased vision. ${ }^{12-14}$ Prior studies of orbital metastases have revealed that $35-61 \%$ of patients had an unknown primary malignancy at the time of their ocular diagnosis. ${ }^{12,13,15}$ In addition, metastasis of cholangiocarcinoma to the globe and orbit is extremely rare. Only two cases have been described previously. ${ }^{10,16}$

To our knowledge, this is the largest case series in the English language ophthalmic literature to describe metastatic cholangiocarcinoma. 


\section{Summary}

\section{What was known before}

- Cholangiocarcinoma is a rare neoplasm that spreads regionally and can metastasize. Cholangiocarcinoma rarely presents with neuro-ophthalmologic manifestations.

\section{What this study adds}

- Five different presentations of neuro-ophthalmologic findings in patients with cholangiocarcinoma. Raise awareness that cholangiocarcinoma can metastasize and can present to ophthalmologists.

\section{Conflict of interest}

The authors declare no conflict of interest.

\section{References}

1 Burak K, Angulo P, Pasha TM, Egan K, Petz J, Lindor KD. Incidence and risk factors for cholangiocarcinoma in primary sclerosing cholangitis. Am J Gastroenterol 2004; 99(3): 523-526.

2 Ghouri Y, Mian I, Blechacz B. Cancer review: cholangiocarcinoma. J Carcinog 2015; 14: 1.

3 Kilbourn K, Aferzon J, Manon M. Isolated brain metastasis in cholangiocarcinoma: a case report and review of literature. Conn Med 2014; 78(3): 161-162.

4 DeOliveira M, Cunningham S, Cameron J, Kamangar F, Winter J, Lillemoe $\mathrm{K}$ et al. Thirty-one-year experience with 564 patients at a single institution. Ann Surg 2007; 245(5): 755-762.
5 Tyson G, El-Serag H. Risk factors of cholangiocarcinoma. Hepatology 2011; 54(1): 173-184.

6 SEER Stat Fact Sheets. Cancer of the Liver and Intrahepatic Bile Duct, 2015. Available at: http://www.seer.cancer.gov.

7 Kitagawa Y, Nagino M, Kamiya J, Uesaka K, Sano T, Yamamoto $\mathrm{H}$ et al. Lymph node metastasis from hilar cholangiocarcinoma: audit of 110 patients who underwent regional and paraaortic node dissection. Ann Surg 2001; 233(3): 385-392.

8 Chindaprasirt J, Sookprasert A, Sawanyawisuth K, Limpawattana P, Tiamkao S. Brain metastases from cholangiocarcinoma: a first case series in Thailand. Asian Pac J Cancer Prev 2012; 13(5): 1995-1997.

9 Mirrhakinov A, Nwanko N, Zdunek T, Bucher N. Cholangiocarcinoma and brain lesions: an extremely rare finding. BMJ Case Rep 2013; e-pub ahead of print 9 May 2013; doi:10.1136/bcr-2013-009235.

10 Fujimoto K, Kuroda J, Makino K, Hasegawa Y, Kuratsu J. Skull metastasis from intrahepatic cholangiocarcinoma: report of 3 cases and review of the literature. Neurol Med Chir (Tokyo) 2013; 53(10): 717-721.

11 Tonn J, Westphal M, Rutka J, Grossman S. Neuro-Oncology of CNS Tumors, 1st ed. Springer: Berlin, 2006, pp 306-308.

12 Amjad A, Osmani AH, Haider G. Proptosis of eye: an atypical presentation of prostatic malignancy. J Coll Physicians Surg Pak 2015; 25(Suppl 1): s39-s40.

13 Char D, Miller T, Kroll S. Orbital metastases: diagnosis and course. Br J Ophthalmol 1997; 81(5): 386-390.

14 Ashton N, Morgan G. Discrete carcinomatous metastases in the extraocular muscles. Br J Ophthalmol 1974; 58(2): 112-117.

15 Font R, Ferry A. Carcinoma metastatic to the eye and orbit III. A clinicopathologic study of 28 cases metastatic to the orbit. Cancer 1976; 38(3): 1326-1335.

16 Miyamoto J, Tatsuzawa K, Sasajima H, Mineura K. Metastatic skull tumor from cholangiocarcinoma-case report. Neurol Med Chir (Tokyo) 2007; 47(3): 132-135. 